\section{Autosomal-dominante Vererbung}

\section{J. Arnemann}

Abteilung Molekulargenetik, Labor Dr. Wisplinghoff, Köln, Deutschland

\section{Synonym(e) AD}

Englischer Begriff autosomal dominant inheritance

Definition Bei autosomal-dominanter Vererbung wird eine autosomale Genvariante so exprimiert, dass sie im Sinne eines Funktionsgewinns die Merkmalsausprägungen anderer Varianten übertrifft, sodass diese nicht oder kaum in Erscheinung treten.

Beschreibung Eine autosomal-dominante Vererbung beschreibt 2 wesentliche Eigenschaften, nämlich eine Zuordnung der Eigenschaft oder der Krankheitsursache auf ein Autosom, d. h. einem Nicht-Geschlechtschromosom mit gleichzeitigem
Ausschluss einer X-chromosomalen Vererbung sowie die Tatsache, dass diese entsprechende Eigenschaft oder auch Krankheitsmutation in der Expression stärker ist als andere und diese in der phänotypischen Ausprägung übertrifft. So ist das Vorhandensein eines dominanten Allels ausreichend, um diese Eigenschaft oder Erkrankung auszuprägen. Treten dominante Krankheitsmutationen homozygot, d. h. in 2 Kopien, auf, so ist der Krankheitsverlauf meist schwerwiegender. Ein typisches Beispiel dafür ist die familiäre Hypercholesterinämie, bei der sich homozygote Patienten oftmals regelmäßig einer Lipidapharese zur extrakorporalen Blutreinigung von LDL-Cholesterin, Lipoproteinen oder Triglyceriden unterziehen müssen.

Der Begriff einer autosomal-dominanten Vererbung ist von Bedeutung in der genetischen Beratung und der formalen genetischen Beschreibung eines Vererbungsmechanismus.

\section{Literatur}

Strachan T, Read AP (2005) Molekulare Humangenetik. Elsevier $\mathrm{GmbH}$, München 